J3eA, Journal sur l'enseignement des sciences et technologies de l'information et des systèmes, Volume 4, Hors-Série 2, 12 (2005)

DOI : http://dx.doi.org/10.1051/bib-j3ea:2005712

(C) EDP Sciences, 2005

Un enseignement d'histoire des sciences et des techniques.

$L$ 'exemple de la naissance de l'automatique française

P. Remaud, P. Bugnet et J.C. Trigeassou

Laboratoire d'Automatique et d'Informatique Industrielle (LAII)

École Supérieure d'Ingénieurs de Poitiers (ESIP)

40 avenue du Recteur Pineau

F-86022 Poitiers CEDEX, France 


\title{
UN ENSEIGNEMENT D'HISTOIRE DES SCIENCES ET DES TECHNIQUES. L'EXEMPLE DE LA NAISSANCE DE L'AUTOMATIQUE FRANCAISE
}

\author{
Patrice Remaud - Pierre Bugnet - Jean-Claude Trigeassou \\ Laboratoire d'Automatique et d'Informatique Industrielle (LAII). Ecole Supérieure \\ d'Ingénieurs de Poitiers (ESIP). 40, Avenue du Recteur Pineau. 86022 POITIERS Cedex \\ Tél : 0549453642 et courrier électronique : patrice.remaud@esip.univ-poitiers.fr
}

\begin{abstract}
Résumé :
L'article proposé s'articule en deux parties. La première a pour objectif de relater notre expérience dans l'enseignement de l'histoire des sciences et des techniques dans le cadre général des sciences de laboratoire (par contraste avec les sciences humaines) au sein des différentes composantes de l'Université de Poitiers. La deuxième partie s'inscrit dans un cadre disciplinaire avec l'histoire de la naissance de l'automatique en France. La rencontre au lendemain de la seconde guerre mondiale entre les membres de l'école de la régulation et des jeunes ingénieurs de l'armement et des télécommunications va aboutir au développement de l'automatique française durant les années 1950.
\end{abstract}

Mots clés : histoire des sciences, histoire des techniques, histoire de l'automatique.

\section{UN ENSEIGNEMENT D'HISTOIRE DES SCIENCES ET DES TECHNIQUES.}

\subsection{Pourquoi?}

Les différentes composantes de l'université de Poitiers ont pris récemment conscience de l'importance d'introduire l'histoire des sciences et des techniques dans leurs cursus d'enseignement. Le principal intérêt de ce type d'enseignement pour un étudiant scientifique est de lui permettre de replacer ses connaissances dans une perspective historique et philosophique. Une expérience est donc menée à l'Université de Poitiers depuis plusieurs années dans le but d'introduire ce type d'enseignement. Il s'appuie sur une recherche en histoire des sciences et des techniques dans le domaine de l'automatique, menée en collaboration entre le Centre d'Histoire Des Techniques du CNAM à Paris et le LAII de l'ESIP à Poitiers. Cette recherche fait l'objet du travail de thèse de Doctorat en Histoire des Sciences et des Techniques de Patrice Remaud. Le titre de cette thèse est Une histoire de la genèse de l'automatique en France.

\subsection{Pour qui ?}

Depuis maintenant quatre années, un enseignement d'histoire des sciences et des techniques est proposé aux étudiants de l'ESIP, de la Faculté des Sciences (DEUG première année par l'intermédiaire d'un cycle de conférences, Licence ès Sciences Physiques, écoles doctorales Sciences pour l'Ingénieur et Ingénierie Chimique Biologique et Géologique), et de différents IUT (Génie Electrique et Informatique Industrielle, Génie Thermique et Energie, Génie Industriel et Maintenance). Par ailleurs, en dehors du secteur scientifique, une expérience devrait être menée l'année prochaine avec un enseignement d'histoire des sciences et des techniques pour des étudiants de Sciences Humaines (Faculté d'Histoire).

\subsection{Sous quelle forme et avec quel volume}

horaire ?

Le volume horaire et le contenu de cet enseignement sont propres à chacune des composantes concernées. Les deux grands types d'intervention sont la conférence et le cours. Pour la conférence, d'une durée de deux heures en DEUG ou de quatre heures à l'IUT, le but est de sensibiliser les étudiants à la matière puis de montrer l'intérêt d'une réflexion centrée sur un questionnement autour des sciences. En ce qui concerne le cours, sa durée dépend de la composante concernée. Elle est de douze heures (six séances de deux heures) pour un étudiant de l'ESIP, de dix huit heures (six séances de trois heures) à l'Ecole doctorale, et de trente heures (quinze séances de deux heures) pour un étudiant de Licence. De manière générale, la séance de deux heures de cours est divisée en deux séquences distinctes. La première heure est consacrée à la présentation d'un thème de la part de l'enseignant, la deuxième est réservée au dialogue avec les étudiants et au travail personnel de l'étudiant sur, par exemple, un texte historique.

\subsection{Et avec quel contenu?}

\subsubsection{Tout d'abord, un questionnement}

De manière générale, l'enseignement débute par deux questions : qu'est-ce qu'une science? qu'est-ce que la méthode scientifique? A partir d'une définition $\mathrm{du}$ mot science empruntée à un dictionnaire, on développe un commentaire autour de termes tels que « connaissances», « universelles », « objet», « méthode », 
《 relations », « objectives », « vérifiées », « preuves.» Puis, on aborde la fameuse «méthode scientifique » utilisée par les scientifiques actuels en précisant les circonstances de son émergence au $\mathrm{XVII}^{\mathrm{e}}$ siècle. La présentation des termes « histoire », « épistémologie » et «philosophie » des sciences s'avère nécessaire. Là encore, on utilise des définitions commentées, puis on relate succinctement l'histoire des grands courants de pensée qui ont animé l'épistémologie et la philosophie des sciences au cours du temps. Ensuite, on présente les grandes problématiques qui ont marqué l'histoire des sciences et des techniques : la science est-elle pure ou impure, le développement de la science s'effectue-t-il de manière interne ou externe, et de manière continue ou discontinue ? En présentant la figure emblématique de l'histoire des sciences et des techniques qu'est Galilée, on peut apporter une réponse à ces trois questions. Le procès de Galilée nous montre les rapports impurs de la science avec l'idéologie religieuse. La légitimité du travail de Galilée cherchée auprès des Princes de Médicis illustre le lien du scientifique avec la société dans laquelle il évolue. L'originalité de la mécanique galiléenne par rapport à la mécanique aristotélicienne apporte un exemple du caractère révolutionnaire de certains travaux scientifiques. Afin que l'étudiant s'approprie quelques repères chronologiques, on présente une synthèse des grands moments de l'histoire des sciences: les sciences babyloniennes, égyptiennes, grecques et arabes, la révolution scientifique du $\mathrm{XVII}^{\mathrm{e}}$ siècle, la révolution industrielle au $\mathrm{XVIII}^{\mathrm{e}}$ siècle puis le formidable progrès des sciences et des techniques au cours des siècles suivants. L'histoire des sciences et des techniques s'est appropriée une dimension sociologique au cours de la deuxième moitié du $\mathrm{XX}^{\mathrm{e}}$ siècle. Ainsi, l'étude de la naissance des institutions scientifiques, universités, académies, écoles d'ingénieurs, expositions, congrès, revues, etc., permet de situer l'évolution du rôle et de la place du scientifique dans la société.

\subsubsection{Un contenu pluridisciplinaire}

Tout d'abord, pour des étudiants scientifiques, il apparaît important de réfléchir aux rôles des mathématiques dans les sciences de laboratoire. L'histoire des mathématiques permet grâce aux développements de la géométrie (en prenant l'exemple des géométries euclidienne et noneuclidienne), de l'algèbre (avec l'histoire de la résolution des équations algébriques) et de l'analyse (avec l'introduction du calcul différentiel et intégral) d'apporter une réponse à beaucoup d'étudiants qui se demandent pourquoi les mathématiques sont au cœur de ce que l'on appelle aujourd'hui les sciences. L'étude de la révolution scientifique aux $\mathrm{XVI}^{\mathrm{e}}$ $\mathrm{XVII}^{\mathrm{e}}$ siècles, avec les figures emblématiques de Galilée et de Newton, permet d'aborder la « révolution de pensée » qui se produisit entre les aristotéliciens et les tenants de la nouvelle méthode scientifique. Cette étude a aussi pour intérêt de positionner dans le temps et de justifier la méthode scientifique que nous pratiquons aujourd'hui. Pour mettre en évidence les liens entre technique et science, l'histoire de l'évolution de la machine à vapeur en Angleterre au XVIII ${ }^{\mathrm{e}}$ siècle, à l'origine de la révolution industrielle, est très intéressante. Le travail de Sadi Carnot en France au début du XIX ${ }^{\mathrm{e}}$ siècle, avec sa mise en évidence des deux premiers principes de la thermodynamique, illustre le paramètre humain présent dans la découverte scientifique. Pour ne pas oublier une des grandes révolutions scientifiques et philosophique, on a choisi de traiter l'histoire de la théorie de l'évolution proposée par Charles Darwin en 1859 et le débat qui en découla entre créationnistes et évolutionnistes. L'histoire de la physique au tournant du $\mathrm{XIX}^{\mathrm{e}}$ et du $\mathrm{XX}^{\mathrm{e}}$ siècle et des soit disant « derniers problèmes » à résoudre, permet d'aborder l'histoire de sciences telles que l'électricité et l'optique, et l'émergence des mécaniques relativiste et quantique.

\section{Bibliographie}

Le travail de l'historien des sciences repose sur un travail bibliographique. Pour aborder ce domaine, nous vous proposons quelques ouvrages généraux :

- Pascal Acot, L'histoire des sciences, Paris, PUF, Collection 'Que sais-je ?' n 3495, 1999

- Bruno Jarrosson, Invitation à la philosophie des sciences, Editions du Seuil, Points Sciences, 1992

- Léna Soler, Introduction à l'épistémologie,

Ellipses, 2000

- Colin Ronan, Histoire mondiale des sciences, Editions du Seuil, Points Sciences, 1988 ( 1 ère éd. 1983)

- Bruno Jacomy, Une histoire des techniques, Editions du Seuil, Points Sciences, 1990

ainsi que quelques travaux illustres :

- Aristote, Leçons de physique, Agora Les Classiques Pocket, 1990

- René Descartes, Discours de la méthode, GarnierFlammarion, Paris, 1966

- Galilée, Dialogue sur les deux grands systèmes du monde, Editions du Seuil, Points Sciences, 1992

- Sadi Carnot, Réflexions sur la puissance motrice $d u$ feu, Editions Gabay, 1990

- Charles Darwin, L'origine des espèces, GarnierFlammarion, Paris, 1992

\section{L'EXEMPLE DE LA NAISSANCE DE L'AUTOMATIQUE FRANCAISE.}

A l'image de ces éléments généraux d'histoire des sciences et des techniques, un enseignement de l'histoire de l'électronique, de l'électrotechnique et d'automatique peut lui aussi être envisagé. En rapport avec notre travail de recherche, et dans le domaine de l'histoire de l'automatique, nous nous proposons d'illustrer un moment important de 
l'histoire de l'automatique française : l'émergence de cette science en France après la deuxième guerre mondiale. C'est en effet à l'occasion de diverses manifestations, conférences, congrès et création de revue, qu'au lendemain de la seconde guerre mondiale, l'automatique française s'est constituée en tant que science. Nous avons donc choisi un certain nombre d'événements qui se déroulèrent au cours des années 1947 et 1956 et qui nous paraissent fondateurs. Mais avant de présenter la situation française, nous ferons une brève présentation de l'histoire générale de l'automatique.

\subsection{Aux origines}

Les origines de la régulation, concept fondateur de l'automatique, remontent à l'Antiquité avec les régulations de niveau des clepsydres des mécaniciens de l'Ecole d'Alexandrie. (III ${ }^{\mathrm{e}}$ siècle av. J. C.). Le véritable essor de la régulation coïncide avec le début de l'ère industrielle grâce au régulateur à boules de James Watt (1736-1819). Le régulateur à boules, un des objets techniques les plus connus, est inventé en 1787 et intégré dans la boucle de régulation de vitesse des machines à vapeur à la fin du XVIII ${ }^{\mathrm{e}}$ siècle. La description du comportement temporel de ce mécanisme par des équations différentielles ainsi que les problèmes de stabilité marquent le début du passage de la technique de régulation vers la science automatique. Les problèmes de stabilité des systèmes régulés ont été formulés dans le courant du XIX ${ }^{\mathrm{e}}$ siècle. L'étude de la stabilité du système de régulation d'un télescope utilisé par l'astronome anglais Sir George Bidell Airy (1801-1892) a débouché sur l'élaboration du critère de Edward John Routh (1831-1907) en 1877 [1]. De même, les problèmes rencontrés par Stodola (1859-1942) sur la stabilité des turbines hydrauliques furent soumis à Adolf Hurwitz (18591919) [2] qui développa son propre critère de stabilité formulé de manière matricielle en 1895. Cette époque est principalement dominée par les mécaniciens et l'approche temporelle.

\subsection{Le développement de l'automatique aux Etats Unis}

A la fin du $\mathrm{XIX}^{\mathrm{e}}$ siècle, on assiste au développement des systèmes de télécommunications, d'abord avec le télégraphe, puis le téléphone et enfin la radiodiffusion. L'avancée théorique essentielle de l'automatique est due aux problèmes posés par les communications téléphoniques à longue distance au début du $\mathrm{XX}^{\mathrm{e}}$ siècle. L'aventure du téléphone au sein des Bell Laboratories a servi de cadre au développement des concepts fondamentaux de l'automatique telle que nous l'enseignons aujourd'hui avec les notions de système, d'analyse fréquentielle, de représentation par schéma-blocs. Les travaux de Harold Black (1898-1983) [3], Harry Nyquist (1889-1976) [4] et Hendrik Bode (19051982) [5] en électronique au cours des années 1920 , 1930 et 1940 constituent la base des théories actuelles caractérisées par l'approche fréquentielle.
Jusqu'à une époque relativement récente, les mécaniciens, les thermiciens, les chimistes, les électriciens, les électroniciens pratiquaient leur propre automatique en s'ignorant mutuellement. Grâce aux travaux des Bell Laboratories, une théorie unifiée de l'automatique a réussi à émerger et à s'imposer progressivement dans tous les domaines. Dans cette présentation succincte, nous omettons de développer l'influence de deux écoles continentales de la régulation que sont les écoles allemande et russe.

\subsection{La situation française}

Alors qu'aux Etats Unis et en Angleterre, le développement des télécommunications durant les années 1930 puis l'effort de guerre durant la deuxième guerre mondiale vont permettre d'accélérer l'émergence de la théorie des systèmes, la situation française est très différente. Malgré le fait que la France s'illustre très tôt dans le domaine des asservissements mécaniques, en particulier avec le servomoteur de Jean Joseph Léon Farcot (18231908) en 1868 [6], elle reste à l'écart de l'approche fréquentielle prônée par les électroniciens. De plus, elle se retrouve isolée durant la seconde guerre mondiale. A la libération, l'introduction des idées nouvelles venant d'outre atlantique fut lente et difficile. Elle se fit au cours de congrès qui se déroulèrent d'abord à l'étranger, le premier eut lieu à Londres en 1947, puis en France, lors du premier congrès d'automatique organisé à Paris en 1956. Il y eut des cycles de conférences en 1945 puis en 1947 au CNAM à Paris. L'année 1956 vit la création de la revue Automatisme qui allait permettre la diffusion des idées nouvelles. Cette année 1956 vit également la naissance d'une association regroupant les automaticiens français, l'Association Française de Régulation et d'Automatisme (A.F.R.A.). Nous avons donc choisi de présenter cinq événements (congrès de Londres et de Paris, cours du CNAM de 1947, création de la revue Automatisme et de l'AFRA) et les principaux personnages y ayant participé.

\subsection{Les grands moments et les personnages \\ 2.4.1. Le congrès de Londres en mai 1947}

Les ingénieurs G. Lehman, ingénieur-conseil auprès de l'Office National d'Etudes et de Recherches Aéronautiques et du Laboratoire Central des Télécommunications, ingénieur Polytechnicien de formation, P. Naslin, ingénieur de l'armement et aussi ingénieur Polytechnicien de formation, J. Loeb, ingénieur en chef des P.T.T., chef de la division Télécommande du C.N.E.T., ainsi que M. Moreau, attaché au C.N.E.T. de Londres, ont participé en mai 1947 au congrès organisé à Londres par l'I.E.E. sur le thème des Régulateurs automatiques et servomécanismes. Toutes ces personnes ont assisté à la première tentative, selon les propres termes de Loeb, «de confronter les points de vue des différentes personnes qui ont à s'occuper des servomécanismes. " Le congrès 
comptait presque uniquement des ingénieurs anglais, un petit nombre d'observateurs étrangers outre la délégation française, un observateur américain et un colonel de l'ambassade soviétique. Ce congrès déboucha sur la publication de trois articles dans la revue L'onde électrique au cours de l'année 1948. $\mathrm{Ce}$ congrès et ces articles furent un moment très important pour l'émergence des idées nouvelles en automatique en France.

\subsubsection{Les conférences du CNAM d'octobre-} novembre 1947

C'est lors de cette année 1947, qui a vu l'organisation du congrès de Londres en mai, que va avoir lieu en France un cycle de conférences réunissant des scientifiques travaillant sur les servomécanismes. Cette série de conférences va avoir une importance majeure dans l'essor de l'automatique en France. C'est A. Métral, professeur au C.N.A.M. et à l'Ecole Supérieure de l'Armement, titulaire de la Chaire de Mécanique au C.N.A.M., qui va organiser, avec le concours des Conseils de Perfectionnement et d'Administration du C.N.A.M. dans le cadre des Conférences d'actualités scientifiques et techniques, une série de conférences sous le titre général Analyse, synthèse et position actuelle de la question des servomécanismes. Les cinq intervenants seront P. Colombani, Ingénieur de l'Air et Ingénieur diplômé des Télécommunications, G. Lehman, professeur à l'Ecole Supérieure d'Electricité et chargé de conférences à l'Ecole Centrale des Arts et Manufactures, J. Loeb, chef de la Division 'Télécommandes' au C.N.E.T., A. Pommellet, Ingénieur en chef du Génie Maritime et professeur à l'Ecole Nationale Supérieure du Génie Maritime et de l'Armement, F. H. Raymond, Docteur de l'Université de Paris, assistant au C.N.A.M. et maître de conférences à l'Ecole Nationale Supérieure de l'Armement.

2.4.3. La création de la revue Automatisme en janvier 1956 et de l'association A.F.R.A. en avril 1956

Le général P. Nicolau est visiblement un personnage central durant les années 1940-1950 dans le développement de l'automatique en France. On le retrouve à l'origine des premiers cours d'automatique au sein de l'Institut Supérieur des Matériaux et de la Construction Mécanique et il est membre du comité de rédaction de la revue Automatisme dès sa création en janvier 1956, puis il en devient le rédacteur en chef en janvier 1958. Cette revue est la première en France à traiter uniquement de problèmes d'automatique. On peut noter que le titre de cette revue n'est pas le mot automatique; ceci illustre les polémiques qui ont animé cette époque autour des termes à employer dans cette nouvelle science. Dans le numéro d'avril 1956 de cette revue, un petit texte au bas d'une page intitulé Création d'une association française de régulation et d'automatisme (A.F.R.A.) signale la naissance de cette association qui a pour but de
« regrouper les efforts épars des ingénieurs, en vue d'approfondir, au cours de réunions amicales, d'une part, les méthodes et les possibilités de l'automatisme, en France, et d'autre part, de donner plus de cohésion à l'action dans le domaine de l'Automatisme. » [7] Donc, la France se dote d'une association dont le président d'honneur est $\mathrm{P}$. Nicolau, le président M. Véron, les vice-présidents V. Broïda et F. Esclangon, le secrétaire général M. Liébault et le trésorier M. J. Vivié. La plupart des membres du bureau sont déjà connus. M. Nicolau, Broïda et Esclangon font partie du Comité de rédaction de la revue Automatisme. M. Véron est à l'origine du cycle de conférences du C.N.A.M. de 1945. P. Nicolau est celui qui a introduit les premiers cours d'automatique en France. Quant à J. Vivié, il est le rédacteur en chef de la revue Mesures-Essais-Régulation-Contrôle Industriel.

2.4.4. Le congrès international de l'automatique de Paris en juin 1956

Pour la première fois en France, du lundi 18 au dimanche 24 juin 1956, s'est déroulé Le Congrès International de l'Automatique de Paris réunissant un millier de participants au C.N.A.M.. Cette initiative en revient à $\mathrm{F}$. H. Raymond qui en assura la présidence. La nécessité de promouvoir l'automatique et ses méthodes, matière et méthodes qui restaient inconnu de la plupart des ingénieurs et chercheurs à cette époque, a poussé F. H. Raymond à trouver le moyen le plus adapté à cette promotion : « Il est donc essentiel qu'un Congrès international établisse un premier bilan des méthodes scientifiques qui s'offrent aux chercheurs de l'automatisme et dresse l'inventaire de ses possibilités techniques. Le congrès de l'automatique aura pour mission, entre autres, de mettre en valeur les théories fondamentales de cette science nouvelle qu'est l'automatique théorique et appliquée - qu'on ne saurait confondre avec la cybernétique dont le caractère doit encore s'affirmer. » [8]

\section{Bibliographie}

1. Edward John Routh, A treatise on the stability of a given state of motion, MacMilan, London, 1877

2. Adolf Hurwitz, On the conditions under which an equation has only roots with negative real parts, Mathematische Annalen, Vol. 46, 1895, pp. 273-284

3. Harold S. Black, Stabilised feedback amplifiers, The Bell System Technical Journal, January, 1934

4. Harry Nyquist, Regeneration theory, Bell System Technical Journal, Vol. 11, 1932, pp. 126-147

5. Hendrik W. Bode, Network analysis and feedback amplifier design, D. Van Nostrand New-York, 1945

6. Joseph Farcot, Le servo-moteur, ou moteur asservi, ses principes constitutifs, variantes diverses, application à la manoeuvre des gouvernails, Paris J. Baudry, 1873

7. Revue Automatisme, avril 1956, n 4, p. 131

8. Revue Automatisme, mars 1956, n 3, p. 109 